\title{
Gene Set Enrichment Analysis(GSEA) of differentially expressed genes in green tea against methionine-choline deficient diet in high-fat patients in the development of non-alcoholic fatty liver disease(NAFLD)
}

\author{
Sarbojoy Saha1, Shampa Barmon²
}

1. Department of Biochemistry and Microbiology, North South University, Dhaka, Bangladesh.

* Corresponding author. Email: sarbojoy.saha@ northsouth.edu

2. Department of Environmental Science and Management, North South University, Dhaka, Bangladesh.

\begin{abstract}
The most common liver disorder nowadays is non-alcoholic fatty liver disease(NAFLD) and it is a progressive disease that rises in severity from steatosis to nonalcoholic steatohepatitis(NASH), fibrosis and cirrhosis to increase risk of developing hepatocellular carcinoma. It is a cause of great concern as there is an estimated seventy million Americans who are currently affected by NAFLD, and this is expected to only increase because of its association with obesity and diabetes and also a lack of therapies to keep its development and progression in check. In this particular study we performed a gene set enrichment analysis(GSEA) of differentially expressed genes in a green tea against methionine-choline deficient diet in high-fat patients in the development of non-alcoholic fatty liver disease(NAFLD). The downregulated genes were used to perform an enrichment analysis and in the ARCHS4 TFs Coexpression database the most significant gene was found to be KLF5_human_tf_ARCHS4_coexpression. In the ARCHS4 Kinases Coexpression pathway database STYK1_human_kinase_ARCHS4 Coexpression was found to be the most significant gene. And finally for the upregulated genes a similar enrichment analysis was performed and in the humancy database $\gamma$-linolenate biosynthesis_Homo sapiens_PWY-6000 gene was discovered to be the most significant one. This study has used bioinformatics tools and the Enrichr software to perform a comparative analysis of differentially expressed gene sets for high-fat patients having a diet consisting of green tea against a methionine-choline deficient diet. Green tea is known to contain several antioxidants and polyphenols which provide protection against many liver diseases such as non-alcoholic fatty liver disease(NAFLD). The present study simply tries to build awareness of this to the general public and allow them to learn more about certain diets which have protective effects against liver diseases. Hopefully by implementing these in their daily lifestyles the public can gain some form of protection against these types of liver disorders.
\end{abstract}

Keywords: non-alcoholic fatty liver disease, steatosis, NASH, gene set enrichment analysis, green tea, methionine-choline deficient diet, $\gamma$-linolenate biosynthesis_Homo sapiens_PWY-6000 


\section{Introduction}

Nowadays in the developed world, non-alcoholic fatty liver disease(NAFLD) is the most common liver disorder (Sass, Chang, and Chopra 2005). It is a progressive disease that increases in severity from steatosis to nonalcoholic steatohepatitis(NASH), fibrosis and cirrhosis to increase hepatocellular carcinoma risk (Wong et al. 2010). There is an estimated seventy million Americans affected by NAFLD (Angulo 2007) and its prevalence is expected to rise due to its association with obesity and diabetes (Wanless and Lentz 1990), and a lack of therapies to keep its development and progression in check. No validated treatments of NAFLD exists beyond weight loss and weight management. Weight loss intervention have a poor success rate, thereby emphasizing the need to validate alternative strategies which can mitigate the disease progression to NASH (Ayyad and Andersen 2000).

Thought the mechanism leading to NASH remain unclear, the 'two-hit' mechanism is often used to describe its simple etiology (Day and James 1998). The 'first-hit' leading to liver steatosis is caused by obesity, insulin resistance and excess lipid accumulation. Then reactive oxygen species and reactive nitrogen mediated 'second-hits' leads to oxidative and nitrative modifications to lipids and proteins, and liver injury and NASH (Fujita et al. 2010).

Reactive species implicated in the 'second-hit' phase include those that have originated from mitochondria, cytochrome P-450 induction (Passeyre D, Fromenti B, Mansouri A,2010) and nicotinamide adenine dinucleotide phosphate(NADPH) oxidase following inflammatory cell activation. Greater hepatic NADPH oxidase activity increased liver inflammation, injury, lipid peroxidation and fibrogenesis in a diet-induced model of NAFLD (Carmiel-Haggai, Cederbaum, and Nieto 2005). Other proinflammatory enzymes like myeloperoxidase(MPO) and inducible nitrous acid synthase, can also cause hepatocyte damage in NASH. In patients with NASH, higher accumulation of MPO-positive Kupffer was accompanied by the liver injury induced by the MPO$\mathrm{H}_{2} \mathrm{O}_{2}$ system, as proven by greater 3-nitro-Tyrosine(N-Tyr) levels. Similarly, greater liver inducible nitrous oxide synthase in rodents having NASH was paralleled by increased reactive nitrogen species(RNS) and N-Tyr in association with liver injury, inflammation and fibrosis (Fujita K et al,2010).

Green tea has potential for use in new molecular therapies. Green tea, made from the dried leaves of the Camelia Sinensis plant, is a popular beverage that has been consumed for thousands of years, and Traditional Chinese medicine has emphasized its use in disease prevention and control (Suzuki, Miyoshi, and Isemura 2012). Epidemiological studies have discovered that green tea consumption is linked to a lower incidence of liver disease (Ui et al. 2009). This is because of the catechins found in green tea, such as epigallo-catechin-3-gallate(EGCG), a polyphenol found in green tea and green tea extract and is the most bioactive and well-studied component of green tea. EGCG has multiple health benefits (Kim, Yang, and Cho 2009) including the attenuation and prevention of liver fibrosis (Ding et al. 2015). In vitro studies have shown that EGCG can reduce liver fibrosis by inhibiting the proliferation of fibroblasts, reducing collagen distribution and upregulating the mitochondrial respiratory chain (Santamarina et al. 2015). Outside the liver disease spectrum, this catechins can attenuate tumor growth factor B9(TGF-b) (Sriram et al. 2015). 
Also, the catechins EGCG downregulates OPN expression by reducing the half-life of EGCG mRNA, EGCG-mediated mRNA degradation leads to reduced closure of wounds (Zapf et al. 2015).

One probable mechanism for EGCG-mediated OPN mRNA degradation is through microRNA(miRNA)mediated mRNA deca. miR181a targets OPN mRNA and downregulates OPN protein expression leading to a suppression of migration and adhesion in HepG2 cells (Bhattacharya et al. 2010) . To note, the expression of multiple miRNAs (Arola-Arnal and Bladé 2011) is modulated by miRNA (Tsang and Kwok 2010) .In this particular study, we have concentrated on three miRNAs: miR-221, miR-181 and miR-10b,as studies have shown that EGCG modulates the expression of these three miRNAs (Milenkovic et al. 2012). Furthermore, miRBase Target Database showed that three miRNA target OPN mRNA(Yamada et al. 2016) .

The objective of this study is to identify differentially expressed genes from individuals having Non-Alcoholic Fatty Liver Disease to determine the efficacy of green tea on hyperlipidemic Animal Model. This was a comparative study between efficacy of $2 \%$ green tea extract and a methionine-choline deficient diet. In order to do this, gene expression dataset series GSE 77964 was used.

\section{Materials and Methods}

\section{Ethical statement}

For this study, mice were used as animal models instead of human patients. The protocol for this study was approved by the Institutional Animal Care and Use Committee (2012A00000156) at The Ohio State University. All mice were acclimatized to the temperature-, light-, and humiditycontrolled facility for at least 2 weeks until WT and Nrf2-null mice were 11-12 weeks old. No harmful chemicals or substances were used on the mice during or after this study. The mice were kept in spacious, controlled environment with sufficient light, air and water. They were given the diet consisting of green tea and also the methionine-choline deficient diet on a group basis. The author and co-author were fully aware of this and gave their informed consent. There are no conflicts of interest regarding this study.

\section{Finding out the differentially expressed Genes from GSE 77964}

From the NCBI website GEO datasets were searched using the term "green tea and methioninecholine deficient diet', and reference series GSE 77964 was analyzed with GEO2R.

For GEO2R analysis two groups named " $2 \%$ green tea extract" and "methionine-choline deficient diet" were defined. Four samples were included in the green tea extract group and three constituted the other group to each groups. the GEOquery (Davis and Meltzer 2007) and limma R (Smyth n.d.) packages from the Bioconductor project were used, and GEO2R analysis was performed (Smyth 2004). Top 250 differentially expressed genes were found using the Benjamini \& Hochberg (false discovery rate) method (Benjamini and Hochberg 1995) P values were adjusted. 
$\log 2$ transformation to the data was applied. R script used to perform the calculation was obtained from the R script tab.

\section{Enrichment Analysis}

Enrichment analysis of Downregulated genes was carried out using

- ARCHS4 TFs co-expression (Lachmann et al. 2017)

- ARCHS4 Kinases Coexp (Lachmann et al. 2017)

Enrichment analysis of Upregulated genes was carried out using

- Humancyc 2016 (Caspi et al. 2016)

\section{R Script}

\# Version info: R 3.2.3, Biobase 2.30.0, GEOquery 2.40.0, limma 3.26.8

\# R scripts generated Tue Apr 17 06:35:20 EDT 2018

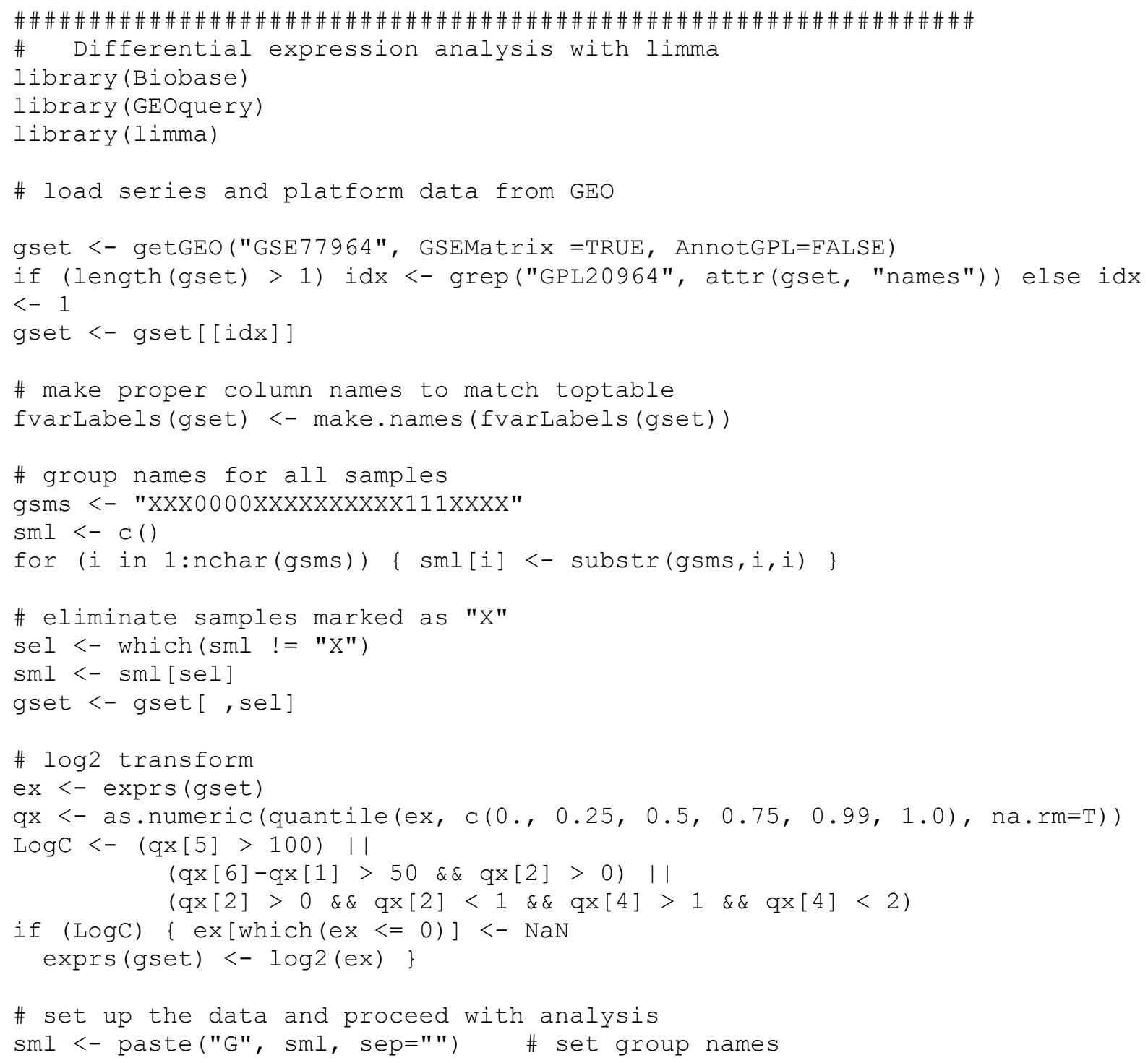




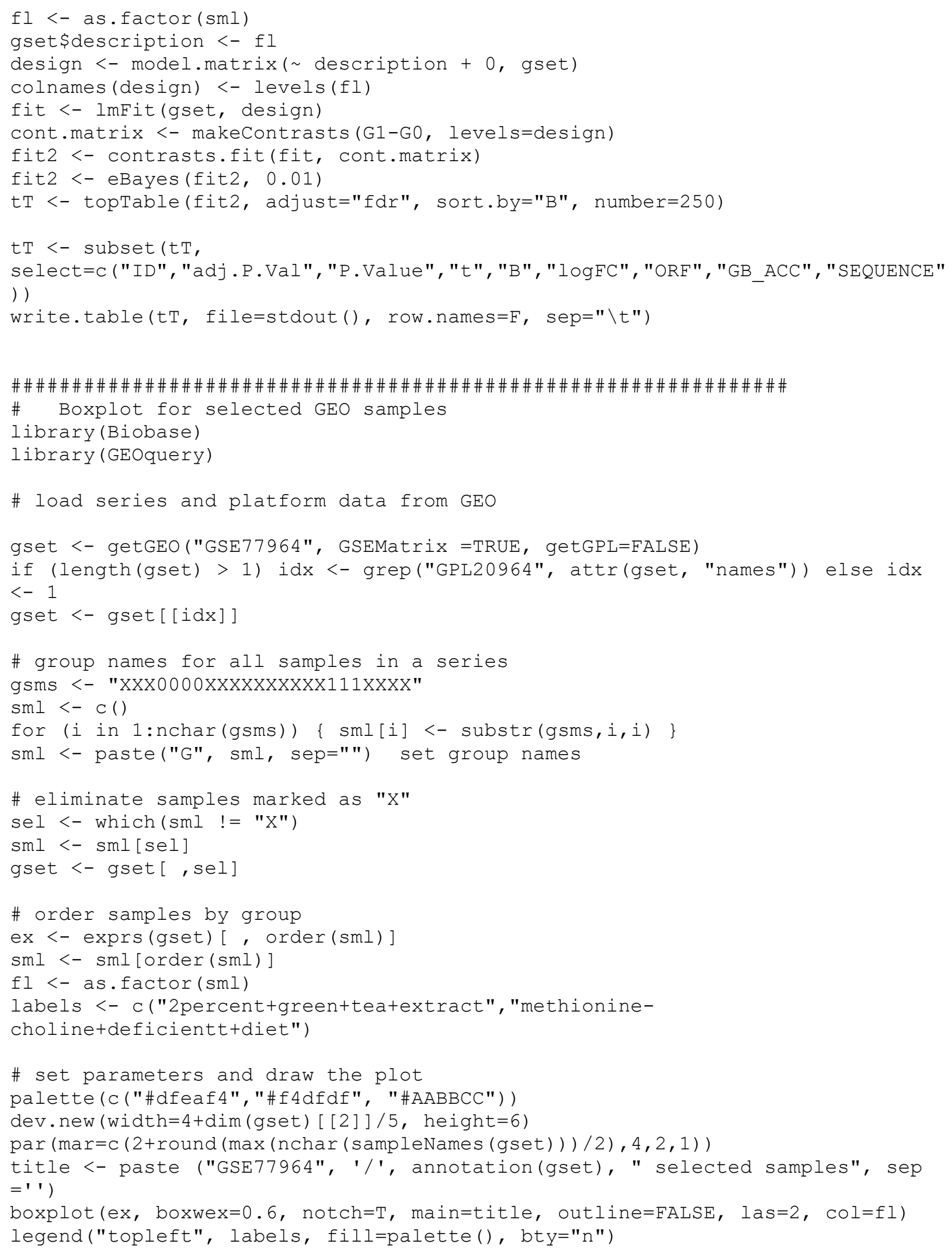




\section{Results and Discussion}

\begin{tabular}{|c|c|c|c|c|c|}
\hline \multicolumn{6}{|c|}{ Downregulated genes } \\
\hline \multicolumn{6}{|c|}{ ARCHS4 TFS Coexp } \\
\hline Index & Name & P-value & $\begin{array}{l}\text { Adjusted } \\
\text { p-value }\end{array}$ & $\begin{array}{l}\text { Z- } \\
\text { scor } \\
\text { e }\end{array}$ & $\begin{array}{l}\text { Combin } \\
\text { ed score }\end{array}$ \\
\hline 1 & $\begin{array}{l}\text { KLF5_human_tf_ARCHS4_coexpressi } \\
\text { on }\end{array}$ & $9.51 \mathrm{E}-09$ & 0.00001 & -1.6 & 29.5 \\
\hline 2 & $\begin{array}{l}\text { ATOH1_human_tf_ARCHS4_coexpre } \\
\text { ssion }\end{array}$ & 7.87E-08 & $\begin{array}{l}0.000016 \\
57\end{array}$ & $\begin{array}{l}- \\
1.6 \\
3\end{array}$ & 26.69 \\
\hline 3 & $\begin{array}{l}\text { TBX10_human_tf_ARCHS4_coexpres } \\
\text { sion }\end{array}$ & 7.87E-08 & $\begin{array}{l}0.000016 \\
57\end{array}$ & $\begin{array}{l}- \\
1.6 \\
2\end{array}$ & 26.43 \\
\hline 4 & $\begin{array}{l}\text { CDX2_human_tf_ARCHS4_coexpress } \\
\text { ion }\end{array}$ & 7.87E-08 & $\begin{array}{l}0.000016 \\
57\end{array}$ & $\begin{array}{l}- \\
1.5 \\
6\end{array}$ & 25.54 \\
\hline 5 & $\begin{array}{l}\text { ISX_human_tf_ARCHS4_coexpressio } \\
\text { n }\end{array}$ & 7.87E-08 & $\begin{array}{l}0.000016 \\
57\end{array}$ & $\begin{array}{l} \\
1.5 \\
4\end{array}$ & 25.18 \\
\hline \multicolumn{6}{|c|}{ ARCHS4 Kinases Coexp } \\
\hline Index & Name & P-value & $\begin{array}{l}\text { Adjusted } \\
\text { p-value }\end{array}$ & $\begin{array}{l}\text { Z- } \\
\text { scor } \\
\text { e }\end{array}$ & $\begin{array}{l}\text { Combin } \\
\text { ed score }\end{array}$ \\
\hline 1 & $\begin{array}{l}\text { STYK1_human_kinase_ARCHS4_coe } \\
\text { xpression }\end{array}$ & 7.87E-08 & $\begin{array}{l}0.000027 \\
08\end{array}$ & $\begin{array}{l} \\
1.6 \\
9\end{array}$ & 27.67 \\
\hline 2 & $\begin{array}{l}\text { MST1R_human_kinase_ARCHS4_coe } \\
\text { xpression }\end{array}$ & $\begin{array}{l}0.000026 \\
33\end{array}$ & 0.004529 & $\begin{array}{l}- \\
1.6 \\
3 \\
\end{array}$ & 17.17 \\
\hline 3 & $\begin{array}{l}\text { EPHB3_human_kinase_ARCHS4_coe } \\
\text { xpression }\end{array}$ & 0.00015 & 0.01032 & $\begin{array}{l} \\
1.6 \\
7 \\
\end{array}$ & 14.71 \\
\hline 4 & $\begin{array}{l}\text { ERBB3_human_kinase_ARCHS4_coe } \\
\text { xpression }\end{array}$ & 0.00015 & 0.01032 & $\begin{array}{l}- \\
1.6 \\
7\end{array}$ & 14.7 \\
\hline 5 & $\begin{array}{l}\text { PTK6_human_kinase_ARCHS4_coexp } \\
\text { ression }\end{array}$ & 0.00015 & 0.01032 & $\begin{array}{l}- \\
1.6 \\
4\end{array}$ & 14.43 \\
\hline \multicolumn{6}{|c|}{ Upregulated genes } \\
\hline $\begin{array}{l}\text { Humancyc } \\
2016\end{array}$ & 0 & 0 & 0 & 0 & 0 \\
\hline Index & Name & P-value & $\begin{array}{l}\text { Adjusted } \\
\text { p-value }\end{array}$ & $\begin{array}{l}\text { Z- } \\
\text { scor } \\
\text { e }\end{array}$ & $\begin{array}{l}\text { Combin } \\
\text { ed score }\end{array}$ \\
\hline
\end{tabular}




\begin{tabular}{|c|c|c|c|c|c|}
\hline 1 & $\begin{array}{l}\gamma \text {-linolenate biosynthesis_Homo } \\
\text { sapiens_PWY-6000 }\end{array}$ & 0.001226 & 0.003679 & $\begin{array}{l}- \\
1.7 \\
4\end{array}$ & 11.67 \\
\hline 2 & $\begin{array}{l}\text { icosapentaenoate biosynthesis II } \\
\text { (metazoa)_Homo sapiens_PWY-7049 }\end{array}$ & 0.001054 & 0.003679 & $\begin{array}{l}- \\
1.6 \\
5\end{array}$ & 11.31 \\
\hline 3 & $\begin{array}{l}\text { bile acid biosynthesis, neutral } \\
\text { pathway_Homo sapiens_PWY-6061 }\end{array}$ & 0.05126 & 0.05126 & -0.3 & 0.88 \\
\hline 4 & $\begin{array}{l}\text { superpathway of choline degradation to } \\
\text { L-serine_Homo sapiens_PWY66-414 }\end{array}$ & 0.02596 & 0.03115 & $\begin{array}{l}- \\
0.1 \\
4\end{array}$ & 0.53 \\
\hline 5 & $\begin{array}{l}\text { pyrimidine ribonucleosides } \\
\text { degradation_Homo sapiens_PWY- } \\
7209\end{array}$ & 0.02229 & 0.03115 & $\begin{array}{l}- \\
0.0 \\
9\end{array}$ & 0.33 \\
\hline
\end{tabular}

The downregulated genes were used to conduct an enrichment analysis and in the ARCHS4 TFs Coexpression database KLF5_human_tf_ARCHS4_coexpression was found to be the most significant gene. And again for these genes in the ARCHS4 Kinases Coexpresion pathway database STYK1_human_kinase_ARCHS4_coexpression was found to be the most significant gene. And for the upregulated genes a similar enrichment analysis was performed, and in the humancy database $\gamma$-linolenate biosynthesis_Homo sapiens_PWY-6000 was discovered to be the most significant pathway. Naturally, this is so as gamma-linoleic acid is a fatty acid which is essential for humans. And so its pathway is also very significant as can be seen from the results from the Humancy database. Some other pathways are also shown in the database as can be seen here.

Gamma linoleic acid(GLA) is a polyunsaturated (18:3) $\omega-6$ fatty acid (the 6 refers to the position of the first double bond from the methyl end of the fatty acid). It is synthesized from linoleic acid by delta ${ }^{6}$ desaturase enzymes. Unlike its precursor, GLA is not an essential fatty acid, since humans possess a delta ${ }^{6}$ desaturase enzyme. GLA is of major importance, being the precursor for the synthesis of several delta ${ }^{6}$-desaturated fatty acids, such as arachidonic acid and eicosapantaenoic acid(EPA). Delta ${ }^{6}$-desaturated fatty acids have roles in the maintenance of membrane structure and function, in the regulation of cholesterol synthesis and transport, in the prevention of water loss from the skin, and as precursors of eicosanoids, including prostaglandins and leukotrienes. Although humans possess a delta ${ }^{6}$-desaturase, they lack a delta ${ }^{12}$ and delta ${ }^{15}$ desaturases, and therefore linoleic acid, the precursor for gamma linoleic acid biosynthesis, is an essential fatty acid that must be obtained through the $\operatorname{diet}($ Fan and Chapkin 1998).

\section{Conclusion}

In this particular study an enrichment analysis was performed for the differentially expressed genes and in the ARCHS4 TFs Coexpression database the most significant gene was found to be KLF5_human_tf_ARCHS4_coexpression. In the ARCHS4 Kinases Coexpression pathway database STYK1_human_kinase_ARCHS4_coexpression was found to be the most significant gene. And finally for the upregulated genes a similar enrichment analysis was performed and in 
the humancy database $\gamma$-linolenate biosynthesis_Homo sapiens_PWY-6000 gene was discovered to be the most significant one. And we know that gamma-linolenate or gamma linoleic acid(GLA) is an essential omega 6 fatty acid, synthesized from linoleic acid, itself another essential fatty acid. It is also a precursor for several other essential fatty acids such as arachidonic acid and eicosapentaenoic acid. It must be obtained from the diet, as humans lack the delta- 6 detasaturase enzymes needed for its synthesis. This study hopes to build some awareness about the prevalence of liver diseases in recent years and the protective measures to counteract this growing problem. This can be in the form of a healthy and balanced diet consisting of healthy options such as green tea which is explained in this study. Hopefully, the common public will become more aware of this increasing concern of non-alcoholic fatty liver disease(NAFLD) and take necessary steps to combat this problem. 


\section{REFERENCES}

Angulo, Paul. 2007. "Obesity and Nonalcoholic Fatty Liver Disease.” Nutrition Reviews 65 (6 Pt 2): S57-63. http://www.ncbi.nlm.nih.gov/pubmed/17605315.

Arola-Arnal, Anna, and Cinta Bladé. 2011. "Proanthocyanidins Modulate MicroRNA Expression in Human HepG2 Cells." Edited by Brian P. Chadwick. PLoS ONE 6 (10): e25982. https://doi.org/10.1371/journal.pone.0025982.

Ayyad, C, and T Andersen. 2000. "Long-Term Efficacy of Dietary Treatment of Obesity: A Systematic Review of Studies Published between 1931 and 1999." Obesity Reviews : An Official Journal of the International Association for the Study of Obesity 1 (2): 113-19. http://www.ncbi.nlm.nih.gov/pubmed/12119984.

Benjamini, Yoav, and Yosef Hochberg. 1995. "Controlling the False Discovery Rate: A Practical and Powerful Approach to Multiple Testing." Journal of the Royal Statistical Society. Series B (Methodological). WileyRoyal Statistical Society. https://doi.org/10.2307/2346101.

Bhattacharya, Syamal D., Juline Garrison, Hongtao Guo, Zhiyong Mi, Jovan Markovic, Victoria M. Kim, and Paul C. Kuo. 2010. "Micro-RNA-181a Regulates Osteopontin-Dependent Metastatic Function in Hepatocellular Cancer Cell Lines." Surgery 148 (2): 291-97. https://doi.org/10.1016/j.surg.2010.05.007.

Carmiel-Haggai, Michal, Arthur I. Cederbaum, and Natalia Nieto. 2005. "A High-Fat Diet Leads to the Progression of Non-Alcoholic Fatty Liver Disease in Obese Rats." The FASEB Journal 19 (1): 136-38. https://doi.org/10.1096/fj.04-2291fje.

Caspi, Ron, Richard Billington, Luciana Ferrer, Hartmut Foerster, Carol A. Fulcher, Ingrid M. Keseler, Anamika Kothari, et al. 2016. "The MetaCyc Database of Metabolic Pathways and Enzymes and the BioCyc Collection of Pathway/Genome Databases." Nucleic Acids Research 44 (D1). Oxford University Press: D471-80. https://doi.org/10.1093/nar/gkv1164.

Davis, S., and P. S. Meltzer. 2007. "GEOquery: A Bridge between the Gene Expression Omnibus (GEO) and BioConductor.” Bioinformatics $23 \quad$ (14): 1846-47. https://doi.org/10.1093/bioinformatics/btm254.

Day, Christopher P., and Oliver F.W. James. 1998. "Steatohepatitis: A Tale of Two 'Hits'?" Gastroenterology 114 (4). Elsevier: 842-45. https://doi.org/10.1016/S0016-5085(98)705992.

Ding, Yi, Xin Sun, Yuning Chen, Yue Deng, and Ke Qian. 2015. "Epigallocatechin Gallate Attenuated Non-Alcoholic Steatohepatitis Induced by Methionine- and Choline-Deficient Diet." European Journal of Pharmacology 761 (August): 405-12. https://doi.org/10.1016/j.ejphar.2015.05.005.

Fan, Yang-Yi, and Robert S. Chapkin. 1998. "Importance of Dietary $\gamma$-Linolenic Acid in Human Health and Nutrition." The Journal of Nutrition 128 (9): 1411-14. https://doi.org/10.1093/jn/128.9.1411.

Fujita, Koji, Yuichi Nozaki, Masato Yoneda, Koichiro Wada, Hirokazu Takahashi, Hiroyuki Kirikoshi, Masahiko Inamori, et al. 2010. "Nitric Oxide Plays a Crucial Role in the 
Development/Progression of Nonalcoholic Steatohepatitis in the Choline-Deficient, 1-Amino Acid-Defined Diet-Fed Rat Model." Alcoholism: Clinical and Experimental Research 34 (February). Wiley/Blackwell (10.1111): S18-24. https://doi.org/10.1111/j.15300277.2008.00756.x.

Kim, Hye-Kyung, Taik-Hoon Yang, and Hong-Yon Cho. 2009. "Antifibrotic Effects of Green Tea on in Vitro and in Vivo Models of Liver Fibrosis." World Journal of Gastroenterology 15 (41): 5200-5205. http://www.ncbi.nlm.nih.gov/pubmed/19891020.

Lachmann, Alexander, Denis Torre, Alexandra B. Keenan, Kathleen M. Jagodnik, Hyojin J. Lee, Moshe C. Silverstein, Lily Wang, and Avi Ma'ayan. 2017. "Massive Mining of Publicly Available RNA-Seq Data from Human and Mouse." BioRxiv, September. Cold Spring Harbor Laboratory, 189092. https://doi.org/10.1101/189092.

Milenkovic, Dragan, Christiane Deval, Erwan Gouranton, Jean-François Landrier, Augustin Scalbert, Christine Morand, and Andrzej Mazur. 2012. "Modulation of MiRNA Expression by Dietary Polyphenols in ApoE Deficient Mice: A New Mechanism of the Action of Polyphenols." Edited by Ralf Krahe. PLoS ONE 7 (1): e29837. https://doi.org/10.1371/journal.pone.0029837.

Santamarina, Aline B., Milena Carvalho-Silva, Lara M. Gomes, Marcos H. Okuda, Aline A. Santana, Emilio L. Streck, Marilia Seelaender, et al. 2015. "Decaffeinated Green Tea Extract Rich in Epigallocatechin-3-Gallate Prevents Fatty Liver Disease by Increased Activities of Mitochondrial Respiratory Chain Complexes in Diet-Induced Obesity Mice." The Journal of Nutritional Biochemistry 26 (11): 1348-56. https://doi.org/10.1016/j.jnutbio.2015.07.002.

Sass, David A, Parke Chang, and Kapil B Chopra. 2005. "Nonalcoholic Fatty Liver Disease: A Clinical Review." Digestive Diseases and Sciences 50 (1): 171-80. http://www.ncbi.nlm.nih.gov/pubmed/15712657.

Smyth, Gordon K. n.d. "Limma: Linear Models for Microarray Data." Accessed April 17, 2018. https://pdfs.semanticscholar.org/b400/022ca3df41788bd020da732746eb7515444e.pdf.

- 2004. "Linear Models and Empirical Bayes Methods for Assessing Differential Expression in Microarray Experiments." Statistical Applications in Genetics and Molecular Biology 3 (1): 1-25. https://doi.org/10.2202/1544-6115.1027.

Sriram, Narayanan, Srinivasan Kalayarasan, Ramar Manikandan, Munusamy Arumugam, and Ganapasam Sudhandiran. 2015. "Epigallocatechin Gallate Attenuates Fibroblast Proliferation and Excessive Collagen Production by Effectively Intervening TGF- $\beta 1$ Signalling." Clinical and Experimental Pharmacology and Physiology 42 (8): 849-59. https://doi.org/10.1111/1440-1681.12428.

Suzuki, Yasuo, Noriyuki Miyoshi, and Mamoru Isemura. 2012. "Health-Promoting Effects of Green Tea." Proceedings of the Japan Academy. Series B, Physical and Biological Sciences 88 (3): 88-101. http://www.ncbi.nlm.nih.gov/pubmed/22450537.

Tsang, Wing Pui, and Tim Tak Kwok. 2010. "Epigallocatechin Gallate Up-Regulation of MiR-16 and Induction of Apoptosis in Human Cancer Cells." The Journal of Nutritional Biochemistry 21 (2): 140-46. https://doi.org/10.1016/j.jnutbio.2008.12.003. 
Ui, Akane, Shinichi Kuriyama, Masako Kakizaki, Toshimasa Sone, Naoki Nakaya, Kaori OhmoriMatsuda, Atsushi Hozawa, Yoshikazu Nishino, and Ichiro Tsuji. 2009. "Green Tea Consumption and the Risk of Liver Cancer in Japan: The Ohsaki Cohort Study." Cancer Causes \& Control 20 (10): 1939-45. https://doi.org/10.1007/s10552-009-9388-x.

Wanless, I R, and J S Lentz. 1990. "Fatty Liver Hepatitis (Steatohepatitis) and Obesity: An Autopsy Study with Analysis of Risk Factors." Hepatology (Baltimore, Md.) 12 (5): 110610. http://www.ncbi.nlm.nih.gov/pubmed/2227807.

Wong, V. W.-S., G. L.-H. Wong, P. C.-L. Choi, A. W.-H. Chan, M. K.-P. Li, H.-Y. Chan, A. M.L. Chim, J. Yu, J. J.-Y. Sung, and H. L.-Y. Chan. 2010. "Disease Progression of NonAlcoholic Fatty Liver Disease: A Prospective Study with Paired Liver Biopsies at 3 Years." Gut 59 (7): 969-74. https://doi.org/10.1136/gut.2009.205088.

Yamada, Shuhei, Shuntaro Tsukamoto, Yuhui Huang, Akiko Makio, Motofumi Kumazoe, Shuya Yamashita, and Hirofumi Tachibana. 2016. "Epigallocatechin-3-O-Gallate up-Regulates MicroRNA-Let-7b Expression by Activating 67-KDa Laminin Receptor Signaling in Melanoma Cells.” Scientific Reports 6 (1): 19225. https://doi.org/10.1038/srep19225.

Zapf, Matthew A.C., Anai N. Kothari, Cynthia E. Weber, Matthew L. Arffa, Phillip Y. Wai, Joseph Driver, Gopal N. Gupta, Paul C. Kuo, and Zhiyong Mi. 2015. "Green Tea Component Epigallocatechin-3-Gallate Decreases Expression of Osteopontin via a Decrease in MRNA Half-Life in Cell Lines of Metastatic Hepatocellular Carcinoma." Surgery 158 (4): 1039-48. https://doi.org/10.1016/j.surg.2015.06.011. 